\title{
Enhanced estimation of loss in the presence of Kerr nonlinearity
}

\author{
Matteo A. C. Rossi ${ }^{*}$ and Francesco Albarelli ${ }^{\dagger}$ \\ Quantum Technology Lab, Dipartimento di Fisica, Università degli Studi di Milano, 20133 Milano, Italy \\ Matteo G. A. Paris ${ }^{\ddagger}$ \\ Quantum Technology Lab, Dipartimento di Fisica, Università degli Studi di Milano, 20133 Milano, Italy; \\ CNISM, Unità Milano Statale, I-20133 Milano, Italy; \\ and INFN, Sezione di Milano, I-20133 Milano, Italy \\ (Received 29 February 2016; published 3 May 2016)
}

\begin{abstract}
We address the characterization of dissipative bosonic channels and show that estimation of the loss rate by Gaussian probes (coherent or squeezed) is improved in the presence of Kerr nonlinearity. In particular, enhancement of precision may be substantial for short interaction time, i.e., for media of moderate size, e.g., biological samples. We analyze in detail the behavior of the quantum Fisher information (QFI), and determine the values of nonlinearity maximizing the QFI as a function of the interaction time and of the parameters of the input signal. We also discuss the precision achievable by photon counting and quadrature measurement and present additional results for truncated, few-photon, probe signals. Finally, we discuss the origin of the precision enhancement, showing that it cannot be linked quantitatively to the non-Gaussianity or the nonclassicality of the interacting probe signal.
\end{abstract}

DOI: 10.1103/PhysRevA.93.053805

\section{INTRODUCTION}

The characterization of quantum channels is a relevant task in quantum technology [1-6]. In particular, characterizing lossy channels in continuous-variable systems is crucial to quantify decoherence [7], to assess quantum illumination protocols [8-11], and to realize quantum reading of classical memories [12]. In some specific cases, the task is simply to discriminate between the presence or the absence of losses [13-15], whereas, in general, a strategy to estimate the exact value of the loss is needed.

The loss rate in optical media and, in turn, the overall loss of the corresponding channels are not observable quantities in a strict sense. As a consequence, one has to infer their value indirectly, i.e., by assessing the influence of loss on a given probing signal by measuring a suitably chosen observable. The overall choice of the probe, of the measurement, and of the data processing is usually referred to as an estimation strategy. Optimization of the estimation strategy, i.e., minimization of intrinsic and extrinsic fluctuations of the estimate, may be pursued upon employing quantum estimation theory [16-19], which provides constructive tools to determine the initial state of the quantum probe and the optimal measurement to be performed at the output. The ultimate bound on precision is set by the quantum Cramér-Rao inequality, written in terms of the so-called quantum Fisher information.

In the past decades, much attention has been devoted to the estimation of loss with different initial preparations of the probes. Optimization over Gaussian input states has been performed [20], showing that ultimate precision may be achieved using photon counting and Gaussian operations at the output. Fock states have also been shown to saturate the ultimate bound on precision $[4,21]$, whereas the performances

\footnotetext{
*matteo.rossi@unimi.it; http://users.unimi.it/aqm

†francesco.albarelli@unimi.it; http://users.unimi.it/aqm

${ }^{\ddagger}$ matteo.paris@ fisica.unimi.it; http://users.unimi.it/aqm
}

of thermal states have been recently investigated [22]. The general scenario of lossy media probed by Gaussian signals at finite temperature has been considered [23], showing that a two-mode squeezed vacuum state is optimal for estimating both the loss parameter and the thermal noise. The benefit of using entanglement in a specific interferometric setup has also been discussed [24]. Recently the problem of estimating both the loss and the phase shift in interferometry has been addressed [25], as well as the related problem of estimating the efficiency of realistic detectors [26,27].

So far, attention has been focused on Gaussian lossy channels where dissipation is due to linear coupling of a radiation mode to the environment, modeled as a bath of external oscillators. On the other hand, optical media where light propagates, such as gasses, biological samples, or optical fibers, may be characterized also by a (usually small) nonlinear response to the electromagnetic field. A question thus arises on whether estimation of linear loss in the presence of nonlinearity is enhanced, or not, compared to the pure linear case. Here, we address this question by considering systems where, besides dissipation due to linear coupling to the environment, some form of nonlinearity is present. In particular, we focus on self-Kerr interaction [28], occurring during propagation of radiation in a nonlinear medium with non-negligible cubic nonlinearity. The Kerr effect has been widely studied in quantum optics either at zero [29] or at finite temperature [30], and attracted interest because it can be employed to generate Schrödinger-cat-like states [31-35]. Nonlinearity of optical fibers has been discussed for its negative impact on the channel capacity [36], whereas its role as a resource in the estimation of losses has not been assessed so far.

As a matter of fact, the presence of nonlinear effects has been already recognized as a resource for quantum estimation, since it allows one to achieve high precision by using robust classical probe states, instead of fragile nonclassical states [37-39]. In particular, Kerr-type nonlinearity may be exploited for estimation of squeezing and displacement of a Gaussian state [40] and to improve Michelson interferometry [41]. 
In this paper, we analyze in detail estimation of loss in the presence of Kerr nonlinearity. We focus mostly on estimation strategies based on Gaussian probes (coherent and squeezed vacuum states), while also briefly examining the use of few-photon probes, the simplest nontrivial ones being optical qutrits. Overall, our results indicate that the presence of Kerr nonlinearity always enhances estimation, improving precision compared to the pure linear case.

In particular, by focusing attention on the estimation of the loss rate parameter of the channel rather than the overall loss (which also includes the interaction time), we make the time dependence explicit. This is a relevant feature of our analysis since dissipation and nonlinearity set two different time scales in the evolution of the probe state. In this way, we address both regimes of "short" and "long" interaction times, showing that (i) nonlinearity always improves estimation and (ii) enhancement of precision may be substantial for short interaction time, i.e., for media of moderate size.

The paper is structured as follows. In Sec. II we briefly review the main tools of quantum estimation theory in order to establish the notation. In Sec. III we present in detail the interaction model we are dealing with, whereas in Sec. IV we discuss the solution of the problem in the absence of nonlinearities. In Sec. V we give an approximate, analytic, solution for the estimation problem with coherent probes, which holds when the Kerr coupling is much smaller than the loss parameter, and present a detailed numerical study for the general case. We also briefly analyze the use of optical qutrit probes and discuss whether non-Gaussianity plays a role in the estimation procedure. Section VI closes the paper with some concluding remarks.

\section{QUANTUM ESTIMATION THEORY}

Here we briefly review local estimation theory and its generalization to quantum systems [18]. In an estimation procedure we want to infer the value of a parameter, say $\gamma$, from the data collected by $n$ measurements, $\left\{x_{1}, \ldots, x_{n}\right\}$. We thus build an estimator $\hat{\gamma}\left(\left\{x_{1}, \ldots, x_{n}\right\}\right)$ that is a function of the outcomes of the measurements. The estimated value of the parameter will be characterized by a statistical error $\delta \gamma$, which is bounded from below by the Cramér-Rao inequality [42]

$$
\delta \gamma^{2} \geqslant \frac{1}{n F(\gamma)}
$$

where $n$ is the size of the sample data (i.e., the number of measurements) and $F(\gamma)$ is the classical Fisher information (FI), defined as

$$
F(\gamma)=\left\langle\left(\frac{\partial \ln p(x \mid \gamma)}{\partial \gamma}\right)^{2}\right\rangle
$$

In Eq. (2) $p(x \mid \gamma)$ is the probability that the outcome of a measurement is $x$ when the value of the parameter is $\gamma$, and $\langle\cdot\rangle$ is the expected value over the probability distribution $p(x \mid \gamma)$.

If the system is quantum, then $p(x \mid \gamma)=\operatorname{Tr}\left(\rho_{\gamma} \Pi_{x}\right)$, where $\rho_{\gamma}$ is the density operator and $\Pi_{x}$ is the POVM operator for the outcome $x$. By introducing the logarithmic symmetric derivative $L_{\gamma}$, satisfying $2 \partial_{\gamma} \rho_{\gamma}=L_{\gamma} \rho_{\gamma}+\rho_{\gamma} L_{\gamma}$, we can rewrite Eq. (2) as

$$
F(\gamma)=\left\langle\frac{\operatorname{Re}\left[\operatorname{Tr}\left(\rho_{\gamma} \Pi_{x} L_{\gamma}\right)\right]^{2}}{\operatorname{Tr}\left(\rho_{\gamma} \Pi_{x}\right)}\right\rangle .
$$

By maximizing $F(\gamma)$ over all possible quantum measurements on the systems we obtain the quantum Fisher information (QFI) $H(\gamma)$, which has the following expression [18]:

$$
H(\gamma)=\operatorname{Tr}\left(\rho_{\gamma} L_{\gamma}^{2}\right)
$$

We can thus write a quantum version of the Cramér-Rao bound,

$$
\delta \gamma^{2} \geqslant \frac{1}{n H(\gamma)}
$$

which gives the ultimate precision achievable on the estimation of $\gamma$ with a quantum measurement. The QFI can be calculated explicitly after a diagonalization of the density operator. Upon writing $\rho_{\gamma}=\sum_{n} p_{n}\left|\psi_{n}\right\rangle\left\langle\psi_{n}\right|$, we get

$$
H(\gamma)=2 \sum_{n, m} \frac{\left|\left\langle\psi_{m}\left|\partial_{\gamma} \rho_{\gamma}\right| \psi_{n}\right\rangle\right|^{2}}{p_{n}+p_{m}},
$$

where the sum is carried out over all $n$ and $m$ such that $p_{n}+$ $p_{m} \neq 0$. If the state of the quantum system is pure, $\rho_{\gamma}=$ $\left|\psi_{\gamma}\right\rangle\left\langle\psi_{\gamma}\right|$, Eq. (6) reduces to

$$
\begin{aligned}
H(\gamma)= & 4\left[\left\langle\partial_{\gamma} \psi_{\gamma} \mid \partial_{\gamma} \psi_{\gamma}\right\rangle+\left\langle\partial_{\gamma} \psi_{\gamma} \mid \psi_{\gamma}\right\rangle^{2}\right. \\
& \left.+\left\langle\psi_{\gamma} \mid \partial_{\gamma} \psi_{\gamma}\right\rangle^{2}+\left|\left\langle\partial_{\gamma} \psi_{\gamma} \mid \psi_{\gamma}\right\rangle\right|^{2}\right] .
\end{aligned}
$$

Notice that the bound in Eq. (5) does depend on the true value of the parameter and, in general (though not necessarily), requires some adaptive or feedback mechanism to be achieved [43]. In other words, in order to exploit local estimation theory one needs some a priori knowledge to roughly locate the value of the parameter. Prior information may come from a theoretical model or some previous experiments, e.g., the measurement of a globally optimized observable [16].

\section{INTERACTION MODEL}

In this work we consider a lossy bosonic channel with a loss rate parameter $\gamma$, which is the quantity that we want to estimate, where nonlinear Kerr effect with coupling $\tilde{\lambda}$ is present. In the absence of any nonlinear effect and working in the interaction picture, the density operator $\rho$ for a single bosonic mode in the channel satisfies a Lindblad master equation of the form

$$
\frac{d \rho}{d t}=\frac{\gamma}{2} \mathcal{L}[a] \rho=\gamma\left(a \rho a^{\dagger}-\frac{1}{2} a^{\dagger} a \rho-\frac{1}{2} \rho a^{\dagger} a\right),
$$

where $a$ is the annihilation operator in the Fock space of the bosonic mode and $\mathcal{L}$ is the Lindblad operator. This equation can be obtained, for instance, from the interaction of the bosonic mode with a bath of harmonic oscillators at zero temperature. The evolution through a Gaussian lossy channel can also be represented as the interaction of the input state with a beam splitter [44], i.e., a bilinear evolution operator $U(\phi)=\exp \left[i \phi\left(a^{\dagger} b+a b^{\dagger}\right)\right]$; the auxiliary mode $b$ is traced out at the end and it is initially in its vacuum state. This picture is connected to the master equation (8) by the relation $\tan ^{2} \phi=e^{\gamma t}-1$; as a matter of fact in previous works [20,21] the estimation of $\gamma$ was recast as the estimation of $\phi$. 
The Kerr interaction is described by a nonlinear term in the Hamiltonian of the system, namely

$$
H_{K}=\tilde{\lambda}\left(a^{\dagger} a\right)^{2} \text {. }
$$

To take into account this effect, the master equation in Eq. (8) now becomes

$$
\frac{d \rho}{d t}=-i\left[H_{K}, \rho\right]+\frac{\gamma}{2} \mathcal{L}[a] \rho .
$$

Upon rescaling the quantities with respect to the loss parameter $\gamma$

$$
\tau=\gamma t, \quad \lambda=\tilde{\lambda} / \gamma
$$

we arrive at

$$
\frac{d \rho}{d \tau}=-i \lambda\left[\left(a^{\dagger} a\right)^{2}, \rho\right]+a \rho a^{\dagger}-\frac{1}{2} a^{\dagger} a \rho-\frac{1}{2} \rho a^{\dagger} a,
$$

which corresponds to the following system of equations for the matrix elements of $\rho$ in the Fock basis:

$$
\begin{aligned}
\frac{d \rho_{p, q}}{d \tau}= & -\left[i \lambda\left(p^{2}-q^{2}\right)+\frac{1}{2}(p+q)\right] \rho_{p, q} \\
& +\sqrt{(1+p)(1+q)} \rho_{p+1, q+1} .
\end{aligned}
$$

The solution for the $\rho_{p, q}$ can be found easily if the initial state is a coherent state, $\rho_{0}=|\alpha\rangle\langle\alpha|$ [34]. It reads

$$
\begin{aligned}
\rho_{p, q}(\tau)= & \frac{\alpha^{p} \bar{\alpha}^{q}}{\sqrt{p ! q !}} \exp \left\{-\frac{1}{2}(p+q) \Delta \tau\right. \\
& \left.-|\alpha|^{2}\left[1-\frac{1-e^{-\Delta \tau}}{\Delta}\right]\right\},
\end{aligned}
$$

where $\Delta=1+2 i \lambda(p-q)$.

We will also consider the case of a squeezed vacuum initial state $\rho_{0}=|r\rangle\langle r|$, where we restrict to a real squeezing parameter $r$, so that the squeezing operator reads $S(r)=$ $\exp \left(\frac{1}{2} r^{2}\left(a^{\dagger 2}-a^{2}\right)\right)$. The explicit analytical expression of the matrix elements of the solution with this initial state can be found in Refs. [45,46], but the matrix elements are known also for arbitrary initial states [47,48]. Notice that for the lossy channel (i.e., a thermal bath at zero temperature) these analytical expressions of the matrix elements are suitable for a numerical computation of the values of the relevant observables. As a matter of fact it is possible to work in a truncated Hilbert space in the Fock basis, since the loss only drives the system into smaller subspaces; this would not be possible if we considered both loss and noise (i.e., a bath with finite temperature). Notice also that $\rho(\tau)$ is in general a mixed state and cannot be diagonalized explicitly, such that an analytic expression for the quantum Fisher information is not available.

We start our analysis by reviewing the analytic solutions when the Kerr effect is not present (i.e., $\lambda=0$ ), and then discuss approximate and numerical solutions for the general case of $\lambda \neq 0$.

\section{SOLUTION IN THE ABSENCE OF NONLINEAR EFFECTS}

When $\lambda=0$, i.e., the nonlinear effects are absent, the channel is Gaussian and in particular a coherent probe state remains pure and coherent during the evolution:

$$
\left|\psi_{\gamma}(\tau)\right\rangle=\left|\alpha e^{-\frac{1}{2} \tau}\right\rangle .
$$

An analytic expression for the QFI is easily obtained using Eq. (7):

$$
H_{\gamma}^{\mathrm{c}}(\tau)=\frac{\bar{n}}{\gamma^{2}} \tau^{2} e^{-\tau},
$$

while for the squeezed vacuum the solution is [20]

$$
H_{\gamma}^{\mathrm{sv}}(\tau)=\frac{\left(-2 e^{\tau}+e^{2 \tau}+2\right) \tau^{2} \bar{n}}{\gamma^{2}\left(e^{\tau}-1\right)\left(2 e^{\tau} \bar{n}-2 \bar{n}+e^{2 \tau}\right)},
$$

where $\bar{n}=|\alpha|^{2}$ for the coherent state and $\bar{n}=\sinh ^{2} r$ for the squeezed vacuum. We also report the QFI for Fock probe states $|n\rangle$, which is optimal when the mean energy is an integer $(\bar{n}=n)$ :

$$
H_{\gamma}^{\mathrm{F}}(\tau)=\frac{\bar{n} \tau^{2}}{\gamma^{2}\left(e^{\tau}-1\right)} .
$$

Notice that in general the quantum signal-to-noise ratio (QSNR) $\gamma^{2} H_{\gamma}(\tau)$ does not depend on $\gamma$ : this means that the bound on the relative error on the estimation of $\gamma$ is constant.

In Fig. 1 we represent the plots of the QFI for the three probe states; this also sums up previous results [20,21] by showing that for small losses the optimal Gaussian state is the squeezed vacuum, for higher losses a coherent state is better, while a Fock state is optimal for every $\tau$. Moreover, we observe that in general $H_{\gamma}(\tau)$ vanishes for $\tau \gg 1$ and has a global maximum at a certain time $\bar{\tau}$. This means that if one is able to control the interaction time in an experiment, setting it to $\bar{\tau}$ allows for optimal estimation of $\gamma$. In particular for the coherent state the optimal time is $\bar{\tau}=2$, with the following optimal value:

$$
\bar{H}_{\gamma}^{\mathrm{c}}=\frac{4|\alpha|^{2}}{e^{2} \gamma^{2}} \text {. }
$$

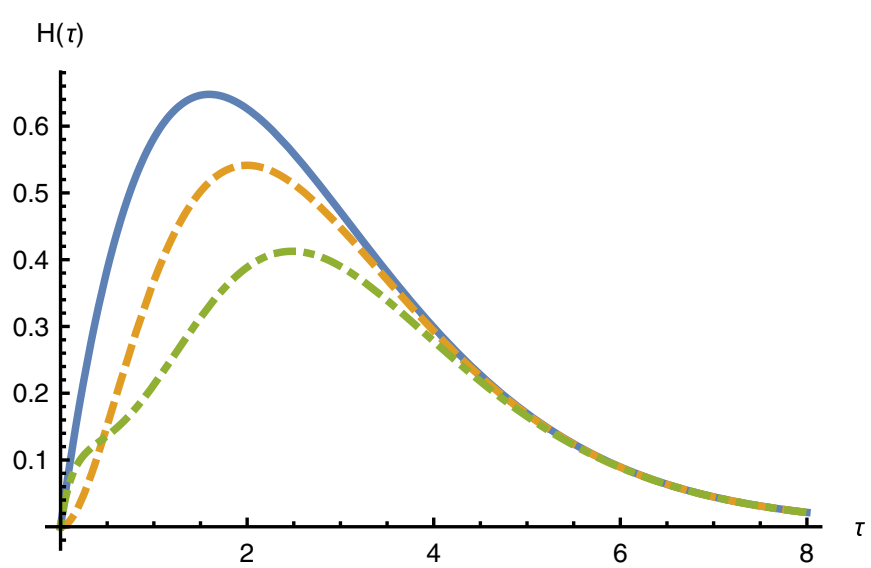

FIG. 1. Plot of the QFI in the absence of nonlinearity as a function of the rescaled time $\tau$ for different probe states at the fixed mean input energy $\bar{n}=1$. The solid blue line represents the optimal Fock state $|1\rangle$, the dashed orange line represents a coherent state, while the dot-dashed green line represents the squeezed vacuum. The graph reflects the general fact that a Fock state is always optimal and for $\tau \rightarrow 0$ the optimal Gaussian state is the squeezed vacuum, while for greater values a coherent state allows for a better estimation. 
As a matter of fact, for coherent states the QFI is saturated by photon number and a quadrature measurement. Let us compute the Fisher information (FI) for these two measurements. The probability distribution for a photon counting experiment for the state is a Poisson distribution with mean $\mu=\left|\alpha e^{-\tau / 2}\right|^{2}$. The FI for a Poissonian is $\mu^{-1}$; hence, using the chain rule of derivatives, we get

$$
F_{n}(\gamma, \tau)=\left(\frac{\partial \tau}{\partial \gamma}\right)^{2}\left(\frac{\partial \mu}{\partial \tau}\right)^{2} \frac{1}{\mu}=\frac{|\alpha|^{2}}{\gamma^{2}} \tau^{2} e^{-\tau} .
$$

The probability distribution for the quadrature measurement $x=\left(a+a^{\dagger}\right) / \sqrt{2}$ is

$$
p(x \mid \gamma)=\left|\left\langle x \mid \alpha e^{-\tau / 2}\right\rangle\right|^{2}=\frac{e^{-\left(x-\sqrt{2} \operatorname{Re}(\alpha) e^{-\tau / 2}\right)^{2}}}{\sqrt{\pi}}
$$

and hence the Fisher information, Eq. (2), is

$$
F_{x}(\gamma, t)=\frac{\tau^{2} e^{-\tau} \operatorname{Re}(\alpha)^{2}}{\gamma^{2}} .
$$

We see that $F_{x}(\gamma, t)=H_{\gamma}(t)$ as long as $\alpha$ is chosen to be real. If $\alpha$ has a complex phase it suffices to choose the proper quadrature or to apply a phase shift to the coherent state to saturate the QFI.

\section{SOLUTION IN THE PRESENCE OF KERR EFFECT}

As stated in Sec. III, with $\lambda \neq 0$ the state $\rho(\tau)$ is a mixed state and not explicitly diagonalizable. In the following, we present an approximate solution for the coherent probe state, valid in the regime of small $\lambda$ and $\tau$, in which the state of the system remains pure and it is thus possible to get an analytical expression for the QFI. Then we show numerical results obtained from a truncation of the Fock space for both coherent and squeezed vacuum probe states. The results are presented both for the optimal time and small time cases; at optimal time only the coherent input is considered since the optimal value of the QFI is always greater than the optimal value of the squeezed vacuum QFI. This fact can be seen in Fig. 2, where we show the behavior of the QFI with and without Kerr interaction for both the Gaussian probes we are considering. From the particular choice of parameters in Fig. 2 we see that the QFI with nonlinear interaction always has a greater value: we will show that this is true in general.

\section{A. Pure state approximation}

When we work with a coherent input state and the nonlinear effect is small compared to the loss parameter, i.e., when $\lambda \ll$ 1 , the state of the system can still be approximated with a pure state for small $\tau$. Expansion of the exponent of $e$ in Eq. (14) to the first order in $\lambda$ and then expansion to the second order of $\tau$ yields

$$
\begin{aligned}
\rho_{p, q}(\tau)= & \frac{\alpha^{p} \bar{\alpha}^{q}}{\sqrt{p ! q !}} \exp \left\{-\frac{1}{2}(p+q) \tau-e^{-\tau}|\alpha|^{2}\right. \\
& \left.-i \lambda\left(p^{2}-q^{2}\right) \tau-i \lambda|\alpha|^{2}(p-q) \tau^{2}\right\} .
\end{aligned}
$$

This is the lowest order of expansion for which we obtain a correction to the quantum Fisher information of Eq. (16).

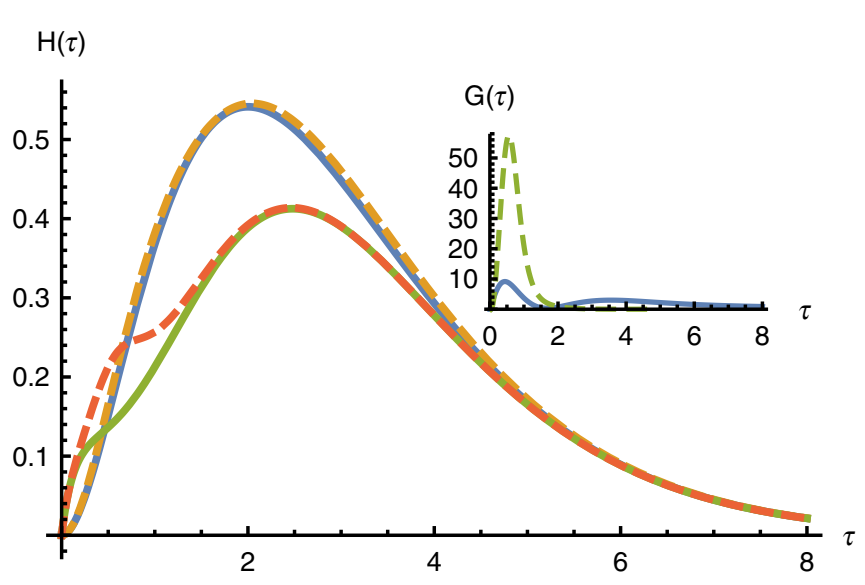

FIG. 2. Plot of the QFI as a function of the rescaled time $\tau$ for different probe states at the fixed mean input energy $\bar{n}=1$. The solid curves are obtained in the absence of nonlinearity, while the dashed curves are obtained in the presence of Kerr nonlinearity (with $\lambda=0.5$ ). The solid blue and dashed orange curves which lie on top in the region $\tau \approx 2$ refer to the coherent state probe, while the solid green and dashed orange curves which lie on top in the region $\tau \approx 0$ refer to the squeezed vacuum probe. In the inset panel we represent the relative gain $G(\tau) \equiv H_{\lambda, \gamma}(\tau) / H_{\gamma}(\tau)-1$ of the QFI in the presence of nonlinearity over the QFI without Kerr effect, shown in percentage. The solid blue line represents the coherent probe, while the dashed green line represents the squeezed vacuum. In both cases there is a peak in gain at $\tau \lesssim 1$, much more pronounced for the squeezed vacuum state. The gain vanishes for increasing $\tau$, but a second, smaller peak can be observed for the coherent state.

The QFI computed for $\rho_{p, q}(\tau)$ of Eq. (23) is

$$
H_{\lambda, \gamma}^{\mathrm{c}}(\tau)=\frac{|\alpha|^{2}}{\gamma^{2}} \tau^{2} e^{-\tau}\left(1+4 \lambda^{2} \tau^{2}|\alpha|^{4}\right)+O\left(\lambda^{3}\right) .
$$

We notice that $H_{\lambda, \gamma}^{\mathrm{c}}(t)$ adds a correction of second order in $\lambda$ and in $\tau$ to $H_{\gamma}^{\mathrm{c}}(\tau)$ of Eq. (16). If we define the relative gain in the estimation of $\gamma$ as $G_{\lambda}(\tau) \equiv H_{\lambda, \gamma}(\tau) / H_{\gamma}(\tau)-1$, then using the pure-state approximation it reads

$$
G_{\lambda}^{\mathrm{c}}(\tau)=4 \lambda^{2} \tau^{2}|\alpha|^{4}+O\left(\lambda^{3}\right) .
$$

The optimal time, up to the second order in $\lambda$, is

$$
\bar{\tau}(\lambda)=2+32 \lambda^{2}|\alpha|^{4}+O\left(\lambda^{3}\right)
$$

and the corresponding optimal QFI is

$$
\bar{H}_{\gamma}^{\mathrm{c}}(\lambda)=\frac{4|\alpha|^{2}}{e^{2} \gamma^{2}}\left(1+16 \lambda^{2}|\alpha|^{4}\right)+O\left(\lambda^{3}\right),
$$

so the optimal relative gain $\bar{G}_{\lambda} \equiv \bar{H}_{\lambda, \gamma} / \bar{H}_{\gamma}-1$ is

$$
\bar{G}_{\lambda}^{\mathrm{c}}=16 \lambda^{2}|\alpha|^{4}+O\left(\lambda^{3}\right) .
$$

Equations (25) and (28) show that the correction to the QFI due to the presence of a small nonlinear effect is positive and increases with $\lambda^{2}$. This means that the nonlinearity of the dispersive medium can be a resource in the estimation of the loss parameter.

The fidelity of the approximate state of Eq. (23) to the exact state (after a truncation of the density matrix) is shown in Fig. 3 as a function of $\tau$ and $\lambda$, for two values of $|\alpha|$. The pure-state 


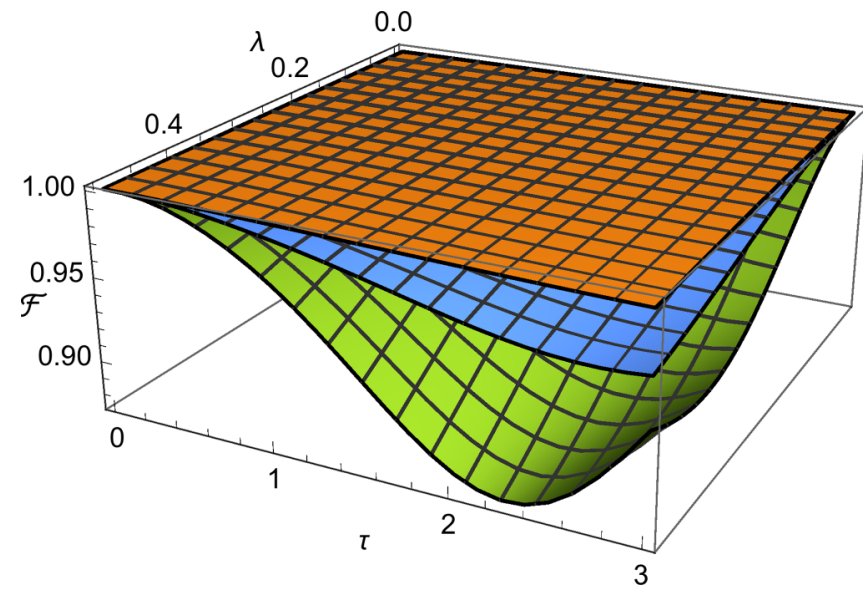

FIG. 3. Fidelity between the pure state of Eq. (23) and the exact state (truncation at 10 photons), for $\alpha=0.5$ (orange), $\alpha=0.75$ (blue), and $\alpha=1$ (green). The fidelity decreases with increasing $\lambda$ and $\alpha$. It temporarily decreases with time, but it tends asymptotically to one as the system reaches the state $|0\rangle$. For small values of $\alpha$ and $\lambda$ the pure-state approximation has fidelity above 0.99 , which then decreases as the energy of the state increases.

approximation is good for a wide range of parameters only if the energy of the initial state is not too big, so that fidelity is close to one $[49,50]$. This means that the analytical expression of the optimal relative gain (28) is good only for small energies, while at a fixed small time $\tau \ll 1$ the relative gain (25) is a good approximation even for higher input energies.

In Sec. V B we calculate the QFI numerically for general values of $\lambda$ and $\alpha$, in order to verify the increase of the QFI also for regions where the pure-state approximation does not hold.

\section{B. Numerical results}

As the density matrix cannot be diagonalized in general and the Fock space is infinite dimensional, in order to evaluate the QFI we resort to numerical diagonalization of the density matrix in a truncated Fock space. The truncation size, which depends on the input energy, is chosen in such a way that the difference between the analytical and the numerical QFI for $\lambda=0$ must be less than $0.001 \%$.

\section{Optimal QFI}

The behavior of the QFI as a function of time for fixed $\lambda$ and $\alpha$ is shown in Fig. 2. The QFI starts from zero and reaches a maximum, then vanishes as $\tau$ increases and the system reaches the zero-photon state $|0\rangle$. Assuming that we are able to control the interaction time of the probe with the channel, we can consider as a figure of merit the optimal QFI, i.e., the maximum of $H_{\lambda, \gamma}(t)$ over time.

In Fig. 4 we show the optimal relative gain in the estimation of $\gamma$. The first notable result is the confirmation of the results obtained in the pure-state approximation: the optimal QFI in the presence of nonlinearity is always greater than without Kerr effect, i.e., the optimal relative gain is always greater than zero. It vanishes for increasing $\alpha$ and $\lambda$ and for $\alpha \rightarrow 0$.

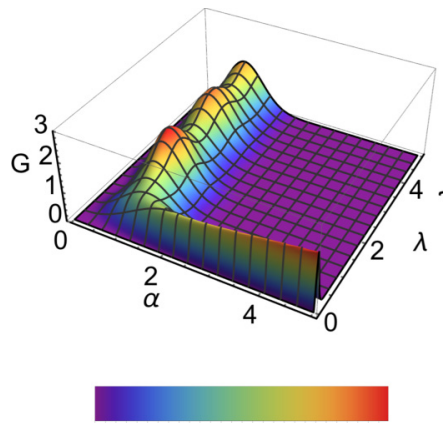

$\begin{array}{llllll}0 & 0.5 & 1.0 & 1.5 & 2.0 & 2.5\end{array}$

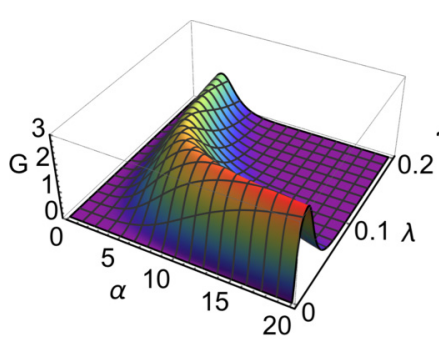

FIG. 4. Optimal relative gain $\bar{G} \equiv \bar{H}_{\lambda, \gamma} / \bar{H}_{\gamma}-1$ of the optimal QFI in the presence of nonlinearity over the optimal QFI without Kerr effect for different regions of $\alpha$ and $\lambda$, shown in percentage. On the left, a 3D plot; on the right, the corresponding contour plot. We can see that the gain is always greater than zero, vanishing for large $\lambda$ and $\alpha$. We can identify two regimes. The first regime, visible in the upper panels when $\alpha \lesssim 2$ is characterized by the presence of local maxima of the gain, which reaches values of about $2 \%$. For large $\lambda$ the improvement reaches a nonvanishing asymptotical value. In the second regime, visible in the lower panels, at fixed $\alpha$ the gain has a single maximum with respect to $\lambda$. As $\alpha$ increases, the maximum moves to smaller values of $\lambda$, but $G$ increases.

By looking at the panels of Fig. 4, we can identify two regimes. The first regime, for $\alpha \lesssim 2$, is characterized by the presence of local maxima of the gain. At fixed $\alpha$, the maxima occur periodically, with $G$ reaching an asymptotic value for $\lambda \rightarrow \infty$. In the second regime, for $\alpha \gtrsim 2$, there is a single local maximum for the gain at fixed $\alpha$. For increasing $\alpha$, the optimal $\lambda$ decreases, but $G$ increases. It is not clear if there is a local maximum for $\alpha$ greater than the values under investigation or if this behavior will persist for $\alpha \rightarrow \infty$, and, in the latter case, if $G$ increases indefinitely or saturates with $\alpha$.

\section{Small time QFI}

Now instead of studying the QFI maximized over time we look at the behavior at a fixed time; in particular we focus on times smaller than the characteristic time of the loss, i.e., $\tau<1$; as an example we study three cases $\tau=0.5,0.1,0.01$. This regime is of interest for media of moderate size, such as biological samples.

In this setting the improvement brought by the nonlinear interaction can be substantial. In Fig. 5 we show the results for a coherent probe state (top row) and for a squeezed vacuum probe state (bottom row). For the squeezed probe we restricted the computation to a smaller range of mean input energies, as 

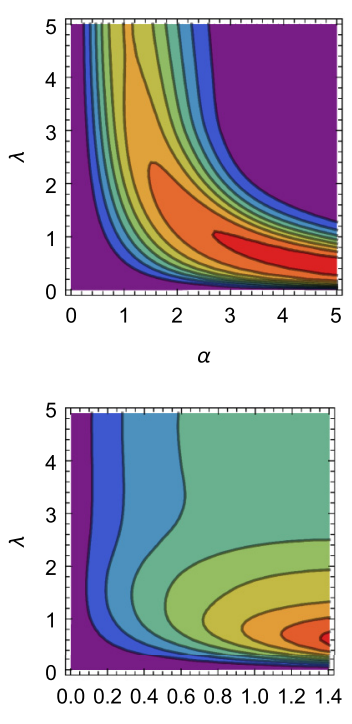

$\bar{n}$
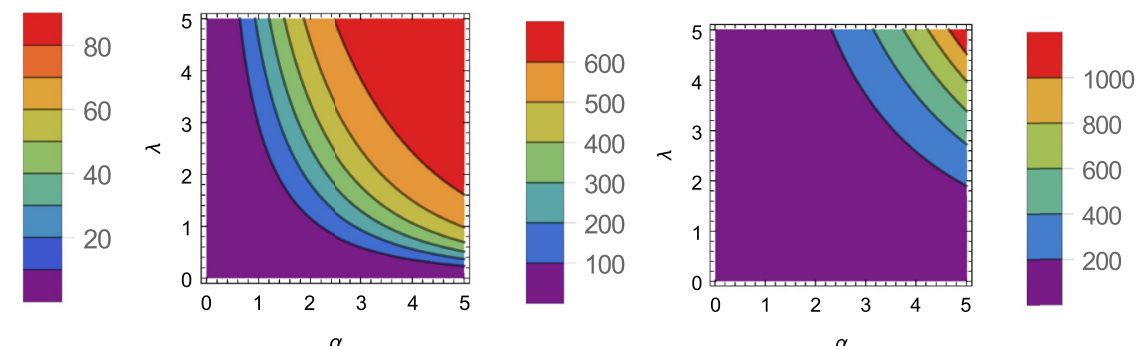

$\alpha$
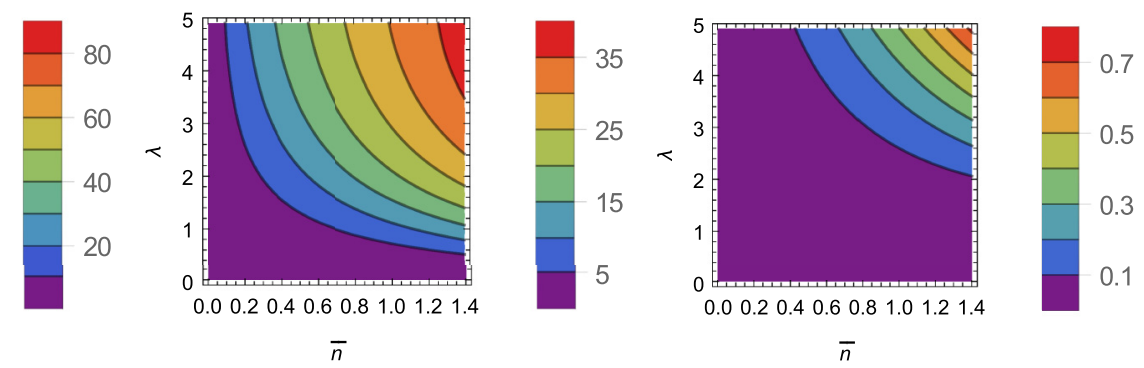

FIG. 5. Relative gain $G(\tau) \equiv H_{\lambda, \gamma}(\tau) / H_{\gamma}(\tau)-1$ of the QFI in the presence of nonlinearity over the QFI without Kerr effect at fixed time for a coherent probe state (top) and for a squeezed vacuum probe state (bottom), shown in percentage. From left to right we have the results for $\tau=0.5,0.1,0.01$. For coherent states we can see a structure similar to that of Fig. 4: the relative gain increases with $\alpha$ and $\lambda$ until it reaches a maximal value, but at small $\tau$ the relative gain is much higher than at the optimal time. For the squeezed vacuum state the gain is smaller as $\tau$ gets smaller (cf. Fig. 2).

the dimension of the truncated Hilbert space needed to obtain a good approximation grows much more rapidly.

By looking at the top-left panel in Fig. 5, the one for $\tau=$ 0.5 , we notice a similar structure to the one in Fig. 4, albeit rescaled. We found that fixing the time parameter $\tau$ changes the scaling in the $\alpha-\lambda$ (or $\bar{n}-\lambda$ ) plane; however, it was not possible to explicitly see this scaling from the analytical expressions of the states.

The improvement due to the Kerr nonlinearity is much more relevant at times which do not correspond to the optimal time, indeed in Fig. 2 we see that the maxima of the graph in the inset panel do not correspond to the ones in the main graph. Moreover, even if the behavior of different input states is slightly different, the most relevant improvement is always obtained for $\tau<1$; this is due to the fact that the value of the QFI at those times is smaller, so that a slight improvement in the absolute value brings a great relative gain.

\section{FI for the quadrature measurement with coherent probe}

Although the optimal QFI is improved by the Kerr effect, we need to find the actual measurement that reaches the quantum bound. In Sec. IV we showed that for a coherent probe both photon counting and quadrature measurement are optimal when $\lambda=0$; however, they are not optimal if the nonlinear term is present. Indeed, photon counting is not affected at all by the Kerr effect, as the diagonal elements of the density matrix are independent of $\lambda$. For this reason we study numerically the effect of nonlinearity on a quadrature measurement. We present the results for a coherent probe state; the analysis is less interesting for a squeezed vacuum probe as the optimal measurement in the linear case is not just a quadrature measurement, but is given by Gaussian operations and photon counting [20].
We found that in general the quadrature measurement is not optimal, i.e., the Fisher information is always lower than the QFI. This fact is presented in the left panel of Fig. 6, for measurements at the optimal time, where the ratio $\bar{R}=$ $F_{x}(\bar{\tau}) / \bar{H}_{\gamma}(\lambda)$ is shown. Here $\bar{H}_{\gamma}(\lambda)$ is the optimal QFI and $F_{x}(\bar{\tau})$ is the FI of the quadrature measurement at the time $\bar{\tau}$ that optimizes the QFI, after an optimization over the quadrature phase (the optimal quadrature phase depends on $\alpha$ and $\lambda$ ). The ratio is close to one only for $\lambda$ close to zero or $\alpha \ll 1$. For increasing $\alpha$ and $\lambda$ the ratio appears to tend asymptotically to $1 / 3$.
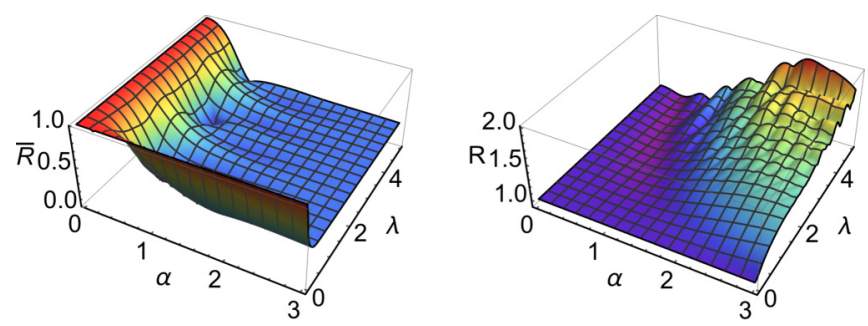

FIG. 6. In the left panel we show the ratio $\bar{R}=F_{x}(\bar{\tau}) / \bar{H}_{\gamma}^{c}(\lambda)$ between the FI of the quadrature at the time $\bar{\tau}, F_{x}(\bar{\tau})$, after an optimization over the quadrature phase and the optimal QFI $\bar{H}_{\gamma}(\lambda)$, for various values of $\lambda$ and $\alpha$. The quadrature measurement is optimal only for $\lambda=0$ and for vanishing energy of the probe $(\alpha \rightarrow 0)$. For $\alpha \lesssim 2$ the ratio oscillates with $\lambda$. For large $\alpha$ and $\lambda$ the ratio reaches asymptotically the value of $1 / 3$. In the right panel we show the ratio $R=F_{x}(\tau) / H_{\gamma}^{c}(\tau)$ for fixed small $\tau=0.1$; the quantity $H_{\gamma}^{c}$ is the QFI without nonlinearities [Eq. (16)]. The quadrature measurement in presence on Kerr effect achieves increasingly better performances for increasing values of $\lambda$ and $\alpha$, even if the ratio has a slightly oscillating behavior and there are some regions in which $R<1$, i.e., the Kerr effect is slightly detrimental. 
In the small time regime a quadrature measurement is still suboptimal in the presence of nonlinearity; however, in some cases such a measurement can perform better than the best possible measurement in the linear case, because the relative improvement of the QFI in this regime is substantial.

In particular, this behavior seems to increase with increasing nonlinearity $\lambda$ and increasing input energy $\alpha$; however, we can see from the right panel of Fig. 6 that oscillations are present and there are small regions where a quadrature measurement does not give an improvement, i.e., $R<1$.

\section{Results with optical qutrit states}

One may wonder what happens if the optimal Fock states are used as probes, instead of Gaussian states.

The obvious answer is that the Kerr nonlinear term $\left(a^{\dagger} a\right)^{2}$ does not affect single Fock states, but also a simple superposition of the form $a|0\rangle+b|n\rangle$ is not affected. The most simple superposition affected by the nonlinear evolution is the optical qutrit state

$$
\cos \theta|0\rangle+e^{i \mu} \sin \theta \sin \varphi|1\rangle+e^{i \nu} \sin \theta \cos \varphi|2\rangle,
$$

where $\theta$ is fixed by choosing the mean energy $\bar{n}$ as the relevant parameter, so that $\theta=\arcsin \sqrt{2 \bar{n} /(3+\cos 2 \varphi)}$.

In the Gaussian lossy evolution, without Kerr nonlinearity, these qutrit states approximate the optimal non-Gaussian states when the mean energy $\bar{n}$ is not an integer; this is particularly important for the low-energy regime $\bar{n}<1$ [21].

In general, the maximum value of the QFI obtainable with the state (29) is the same regardless of the Kerr term in the evolution, but the maximum happens for different values of the initial parameters and at a different time. This is due to the fact that during the evolution the system is constrained to remain in the subspace of dimension three; so if we optimize on every possible parameter there is no room for improvement left.

However, in order to achieve the maximal QFI one should be able to tune the value of the initial parameters for every mean energy $\bar{n}$, and in the nonlinear case also for every value of $\lambda$. In particular in the linear case the result must be optimized only over the parameter $\varphi$, since the relative phases $\mu$ and $v$ give an optimal result for the value $\pi$.

We thus resort to work in a setting similar to the one used to study the optimal gain for the coherent states: given a fixed initial state we check if the nonlinear evolution brings an improvement. In particular, we fix $\mu=\nu=\pi$ and we check the behavior of the quantum Fisher information for different values of $\varphi$, while optimizing over time $t$. The results are in Fig. 7: we find that on average the nonlinear terms bring an improvement for values of $\lambda \approx 1$, i.e., when the nonlinear parameter is approximately equal to the loss parameter to estimate. For higher values of $\lambda$ we have an oscillatory behavior and on average the nonlinearity can also be detrimental.

We also found that at fixed small times the nonlinear Kerr term does not always bring an improvement on average when using qutrit states.
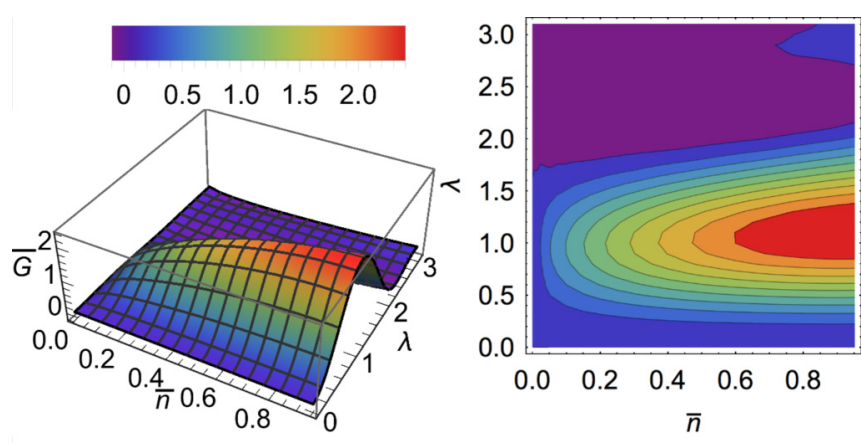

FIG. 7. Average relative gain of the optimal QFI in the presence of nonlinearity over the optimal QFI without Kerr effect for qutrit states, shown in percentage. The range of the parameters are $0<\bar{n}<1$ and $0<\lambda<\pi$. On the left, a 3D plot; on the right, the corresponding contour plot. Every point in the plot is the average improvement obtained by generating 1000 random values of the parameter $\varphi$ of the state (29) in the range $\left(0, \frac{\pi}{2}\right)$, while the phases are fixed $\mu=\nu=\pi$ and $\theta$ is fixed by the choice of the mean energy $\bar{n}$.

\section{Discussion}

The nonlinear Kerr interaction makes the initial Gaussian probe non-Gaussian and nonclassical during the evolution. A question arises on whether the observed increase of the QFI may be quantitatively linked to some quantifier of non-Gaussianity [51] or nonclassicality [52]. Indeed, it would be desirable to identify the proper resource which guarantees the improvement in the estimation by means of a nonlinear interaction, since this would represent a guideline to engineer optimal estimation schemes. On the other hand, also a qualitative indicator to assess the effectiveness of Kerr interaction to enhance precision may be useful.

It has been conjectured [21] that a family of optimal nonGaussian states exists for any fixed energy and, at the same time, that non-Gaussianity in itself cannot be a resource, since there are non-Gaussian states which are far less efficient probes than the optimal Gaussian ones. Our results go in the same direction. In fact, during the evolution described by Eq. (10) a Gaussian input state becomes at first non-Gaussian and then it evolves towards the Gaussian state $|0\rangle$, which is the stationary state. This qualitative behavior is also shown by the relative gain in the estimation of $\gamma$, as can be seen in Fig. 2. These two quantities, however, do not have a quantitative relation in general, e.g., states leading to the largest improvement at optimal time are not the most non-Gaussian.

The picture is unchanged if we instead inspect nonclassicality as a resource, instead of non-Gaussianity. In particular, nonclassicality quantified by the entanglement potential [52] can be directly linked to the presence of coherences (offdiagonal matrix elements) in the basis of Glauber coherent states $[53,54]$. Despite such coherences being very fragile and loss sensitive we have not found any quantitative relation with precision enhancement.

Overall, our results show that while the evolution drives the Gaussian input into a set of non-Gaussian and nonclassical states which are more sensitive to loss detection, neither non-Gaussianity nor nonclassicality can be considered as proper resources. This idea is confirmed by looking at the 
behavior of qutrit probe states, which are already highly non-Gaussian and nonclassical: there we find evidence that the Kerr interaction may be detrimental in some regimes, whereas, when an improvement is present, the states are not necessarily more non-Gaussian or nonclassical.

\section{CONCLUSIONS}

In conclusion, we have addressed the characterization of dissipative bosonic channels in the presence of nonlinearity and shown that the estimation of the loss rate by coherent or squeezed probes is improved in the presence of Kerr nonlinearity. In particular, enhancement of precision may be substantial for short interaction time, i.e., for media of moderate size, whereas for larger media the improvement is asymptotically negligible.

We have analyzed in detail the behavior of the quantum Fisher information (QFI), and have found the values of nonlinearity maximizing the QFI as a function of the interaction time and of the parameters of the input signal. We have also shown that Kerr nonlinearity may be helpful also using few photon probes as optical qutrits.

We have discussed the precision achievable by photon counting and quadrature measurement, showing that they cannot, in general, achieve the QFI in the presence of nonlinearity. On the other hand, for short interaction times even this suboptimal measurement offers a precision improvement compared to the linear case.

Finally, we have discussed the possible origin of the precision enhancement, showing that it cannot be linked quantitatively to the non-Gaussianity and nonclassicality of the interacting probe signal.

\section{ACKNOWLEDGMENTS}

The authors thank Benoit Vallet for his contribution in the early stage of this project. This work has been supported by EU through the Collaborative Project QuProCS (Grant Agreement No. 641277) and by UniMI through the H2020 Transition Grant No. 15-6-3008000-625.
[1] G. M. D’Ariano and P. Lo Presti, Phys. Rev. Lett. 86, 4195 (2001).

[2] A. Fujiwara, Phys. Rev. A 63, 042304 (2001).

[3] G. M. D’Ariano and P. Lo Presti, Phys. Rev. Lett. 91, 047902 (2003).

[4] M. Sarovar and G. J. Milburn, J. Phys. A: Math. Theor. 39, 8487 (2006).

[5] M. Lobino, D. Korystov, C. Kupchak, E. Figueroa, B. C. Sanders, and A. I. Lvovsky, Science 322, 563 (2008).

[6] S. Olivares and M. G. A. Paris, Phys. Rev. A 76, 042120 (2007).

[7] A. Serafini, M. G. A. Paris, F. Illuminati, and S. D. Siena, J. Opt. B 7, R19 (2005).

[8] H. P. Yuen and R. Nair, Phys. Rev. A 80, 023816 (2009).

[9] S.-H. Tan, B. I. Erkmen, V. Giovannetti, S. Guha, S. Lloyd, L. Maccone, S. Pirandola, and J. H. Shapiro, Phys. Rev. Lett. 101, 253601 (2008).

[10] S. Guha and B. I. Erkmen, Phys. Rev. A 80, 052310 (2009).

[11] G. Brida, M. Genovese, and I. Ruo Berchera, Nat. Photon. 4, 227 (2010).

[12] S. Pirandola, Phys. Rev. Lett. 106, 090504 (2011).

[13] M. Sasaki, R. Momose, and O. Hirota, Phys. Rev. A 55, 3222 (1997).

[14] M. G. A. Paris, Phys. Rev. A 64, 014304 (2001).

[15] C. Invernizzi, M. G. A. Paris, and S. Pirandola, Phys. Rev. A 84, 022334 (2011).

[16] C. W. Helstrom, Quantum Detection and Estimation Theory (Academic Press, New York, 1976).

[17] S. L. Braunstein and C. M. Caves, Phys. Rev. Lett. 72, 3439 (1994).

[18] M. G. A. Paris, Int. J. Quant. Inf. 07, 125 (2009).

[19] B. M. Escher, R. L. de Matos Filho, and L. Davidovich, Nat. Phys. 7, 406 (2011).

[20] A. Monras and M. G. A. Paris, Phys. Rev. Lett. 98, 160401 (2007).

[21] G. Adesso, F. Dell'Anno, S. De Siena, F. Illuminati, and L. A. M. Souza, Phys. Rev. A 79, 040305(R) (2009).
[22] G. Spedalieri, S. L. Braunstein, and S. Pirandola, arXiv:1602.05958.

[23] A. Monras and F. Illuminati, Phys. Rev. A 83, 012315 (2011).

[24] H. Venzl and M. Freyberger, Phys. Rev. A 75, 042322 (2007).

[25] P. J. D. Crowley, A. Datta, M. Barbieri, and I. A. Walmsley, Phys. Rev. A 89, 023845 (2014).

[26] M. Barbieri, A. Datta, T. J. Bartley, X.-M. Jin, W. S. Kolthammer, and I. A. Walmsley, arXiv:1502.00681.

[27] S. Grandi, A. Zavatta, M. Bellini, and M. G. A. Paris, arXiv:1505.03297.

[28] R. Boyd, Nonlinear Optics, 3rd ed. (Academic Press, Burlington, MA, 2008).

[29] G. J. Milburn and C. A. Holmes, Phys. Rev. Lett. 56, 2237 (1986).

[30] M. Stobińska, G. J. Milburn, and K. Wódkiewicz, Phys. Rev. A 78, 013810 (2008).

[31] B. Yurke and D. Stoler, Phys. Rev. Lett. 57, 13 (1986).

[32] B. Yurke and D. Stoler, Physica B 151, 298 (1988).

[33] A. Miranowicz, R. Tanas, and S. Kielich, Quantum Opt. 2, 253 (1990).

[34] M. G. A. Paris, J. Opt. B 1, 662 (1999).

[35] H. Jeong, M. S. Kim, T. C. Ralph, and B. S. Ham, Phys. Rev. A 70, 061801 (2004).

[36] R.-J. Essiambre and R. W. Tkach, Proc. IEEE 100, 1035 (2012).

[37] A. Luis, Phys. Lett. A 329, 8 (2004).

[38] Á. Rivas and A. Luis, Phys. Rev. Lett. 105, 010403 (2010).

[39] A. Luis, SPIE Rev. 1, 018006 (2010).

[40] M. G. Genoni, C. Invernizzi, and M. G. A. Paris, Phys. Rev. A 80, 033842 (2009).

[41] A. Luis and Á. Rivas, Phys. Rev. A 92, 022104 (2015).

[42] H. Cramèr, Mathematical Methods of Statistics (Princeton University Press, Princeton, NJ, 1946).

[43] O. E. Barndorff-Nielsen and R. D. Gill, J. Phys. A: Math. Theor. 33, 4481 (2000).

[44] G. M. D’Ariano, Phys. Lett. A 187, 231 (1994). 
[45] G. Milburn, A. Mecozzi, and P. Tombesi, J. Mod. Opt. 36, 1607 (1989).

[46] V. Peřinová and A. Lukš, J. Mod. Opt. 35, 1513 (1988).

[47] V. Peřinová and A. Lukš, Phys. Rev. A 41, 414 (1990).

[48] S. Chaturvedi and V. Srinivasan, J. Mod. Opt. 38, 777 (1991).

[49] M. Bina, A. Mandarino, S. Olivares, and M. G. A. Paris, Phys. Rev. A 89, 012305 (2014).
[50] A. Mandarino, M. Bina, S. Olivares, and M. G. A. Paris, Int. J. Quant. Inf. 12, 1461015 (2014).

[51] M. G. Genoni and M. G. A. Paris, Phys. Rev. A 82, 052341 (2010).

[52] J. K. Asbóth, J. Calsamiglia, and H. Ritsch, Phys. Rev. Lett. 94, 173602 (2005).

[53] W. Vogel and J. Sperling, Phys. Rev. A 89, 052302 (2014).

[54] N. Killoran, F. E. S. Steinhoff, and M. B. Plenio, Phys. Rev. Lett. 116, 080402 (2016). 